\title{
NOTE TECHNIQUE Versants des collines de la ville de Lyon: méthodologie de la prévention des accidents géotechniques
}

\author{
L. VINET, R. KASTNER, P. LARÉAL, N. MONGEREAU \\ Laboratoire Géotechnique, INSA de Lyon*
}

Rev. Franç. Géotech. n64, pp. $67-69$ (juillet 1993)

\section{LES VERSANTS DES COLLINES LYONNAISES}

\subsection{Présentation}

La ville de Lyon est bâtie au confluent du Rhône et de la Saône. Elle compte deux collines principales: La Croix-Rousse et Fourvière, et leurs prolongements. Ces reliefs dominent de 80 à $120 \mathrm{~m}$ le cours des eaux. Les versants sont tantôt subverticaux, en pied de talus, tantôt en pente douce, de 25 à $30^{\circ}$ en partie haute. Ils sont localement appelés « balmes » selon l'usage régional de ce terme qui par ailleurs signifie plutôt grotte, abris sous roche, surplomb rocheux (cf. baume, beaume). Le territoire communal compte des versants très urbanisés, mais une grande partie sont également boisés ou aménagés en terrasses et jardins.

\subsection{Géologie des collines lyonnaises}

Le substratum géologique de ces collines est une «ossature granitique et gneissique parfois affleurante en falaises mais le plus souvent masquée par une couverture sédimentaire. Celle-ci se compose de sables fins, molassiques, et d'argiles graveleuses du Miocène helvétien et tortonien, des argiles et cailloutis villafranchiens, des moraines caillouteuses ou argileuses quaternaires et des loess et limons de couverture. Le socle cristallophyllien est très fracturé et altéré en surface; les sédiments sont compacts mais non consolidés, exceptés quelques grésifications partielles dans les sables molassiques et les cailloutis morainiques (fig. 1).

\subsection{Genèse des versants}

Les balmes résultent d'un talutage naturel dû à l'enfoncement post-glaciaire du réseau hydrographique RhôneSaône. Un tel façonnage, sur un substratum en partie meuble, se traduit par un décapage des pieds de versants qui occasionne un déséquilibre de l'ensemble de la pente. Le rééquilibrage s'effectue par une série de petits glissements qui mettent en place, le long du versant un manteau de colluvions où l'on retrouve les ma-

\section{- 69621 Villeurbanne Cedex.}

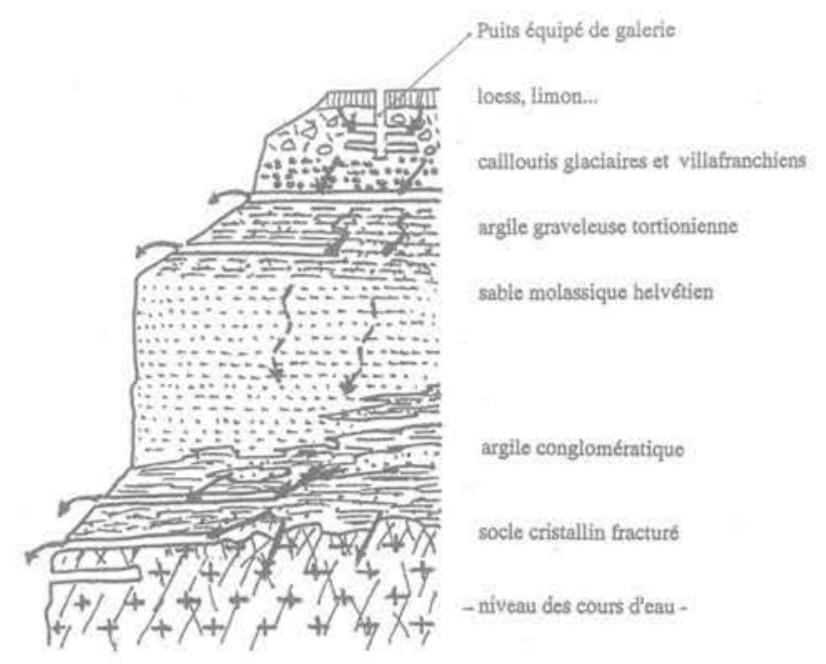

Fig. 1. - Profil synthétique d'un versant de colline

à Lyon (Balme) montrant la succession des horizons géologiques, les circulations d'eau et la position des galeries de captage et de drainage.

- circulation d'eau:

- zones d'émergence:

tériaux constitutifs du haut des collines. Ainsi la géologie des versants résulte-t-elle tantôt d'un affleurement de couches géologiques en place, tantôt de formations superficielles glissées le long des pentes jusqu'à une époque récente (historique).

Certaines entailles de pied de versants sont aussi d'origine anthropique, ainsi que de nombreux aménagements en terrasses fixés par des murs de soutènements. Dans ces circonstances, l'équilibre des versants dépend de l'état des ouvrages réalisés.

\subsection{Hydrogéologie et galeries souterraines}

Le toit du Miocène argilo-graveleux soutient une importante nappe perchée qui produit des sources de trop-plein à mi-pente. Ces sources ont été équipées et développées, depuis l'époque romaine, par des galeries de captage qui s'enfoncent sous terre de quelques dizaines à quelques centaines de mètres. Des galeries mo- 
dernes $(1930,1950)$ ont été creusées afin de drainer les collines et faire disparaitre les suppressions hydrauliques. Enfin, quelques galeries anciennes n'ont pas de rôle hydraulique reconnu (fig. 1).

\section{LES RISQUES GÉOTECHNIQUES}

\subsection{Les risques géotechniques}

Les risques géotechniques identifiés sur le territoire de la commune de Lyon sont liés à des facteurs structurels tels que la pente, la géologie et les états des aménagements humains (soutènements vétustes, galeries effondrées, etc.) et à des facteurs aggravants, tels que des précipitations atmosphériques abondantes, fuite de canalisations d'eau ou travaux imprudents (excavations en pied de mur...).

\subsection{Typologie des accidents potentiels et gravité}

Plusieurs années d'observations ont permis de dresser une typologie des accidents possibles. $\mathrm{Ce}$ sont de classiques glissements de terrain affectant les colluvions de pente, des desquamations dans les talus de sols fins (sable molassique et loess), des coulées boueuses, occasionnées par d'importantes fuites d'eau, des éboulements dans les falaises rocheuses de granite et de gneiss aussi que dans des surplombs de poudingues, des fontis liés à des effondrements en galeries, des ruptures de murs de soutènements et des venues d'eau.

L'importance des dégâts observés n'est pas toujours considérable mais la localisation en site urbanisé leur confère une gravité qui s'est quelquefois avérée catastrophique. Les cubages déplacés sont de quelques mètres cubes à quelques centaines de mètres cubes et les victimes ont été de 40 et 30 en 1930 et 1932 et de trois en 1977.

Quant aux dommages matériels, ils sont souvent tels que des particuliers ne peuvent y faire face.

\section{PRÉVENTION}

\subsection{A travers les âges}

Les habitants des balmes ont cherché à s'affranchir des mouvements de terrain et chutes de matériaux en équipant les pentes de nombreux murs de soutènements ou de placage dont certains de grande hauteur.

\subsection{Depuis 1930 , date de la catastrophe de Fourvière}

En 1930, le maire de l'époque charge une « Commis. sion des Balmes » d'étudier les causes de la catastrophe du mois de novembre ( 30 morts) et, bientôt, d'étudier les conditions de stabilité des versants des collines de la ville.

Vers 1950 , une première cartographie des zones menacées voit le jour pour la colline de la Croix-Rousse.

\subsection{Depuis 1977}

En 1977, la Commission est réactivée, et en 1979 s'amorce une politique de visites systématiques des zones à risque dont une cartographie est dressée en 1982 pour toute la commune: zones rouges à risque géologique fort et zones oranges à risque géologique atténué.

Depuis, parallèlement au traitement des accidents, la Commission effectue de la prévention par l'examen des permis de construire et par des visites systématiques sur le terrain.

Les permis de construire des opérations situées en zones rouges et oranges sont examinés par la Commis. sion après leur obtention: elle donne son avis sur le principe des fondations et le respect des conditions de stabilité d'ensemble du versant. Les points examinés sont les suivants:

- drainage des parties enterrées et conservation des écoulements ;

- adaptation des fondations aux caractéristiques du terrain ;

- satisfaction de la structure à la «condition de fontís »: qu'elle soit calculée pour résister à l'apparition inopinée d'une cavité souterraine de $2 \mathrm{~m}^{2}$ en quelque point que ce soit du sous-sol;

- sauvegarde de la stabilité des mitoyens, terrains ou constructions et de l'ensemble du versant.

Les visites se font systématiquement par secteurs situés en zones rouges et oranges: terrain et bâtiments sont décrits, les renseignements géologiques et hydrogéologiques sont collectés, et les indices ou facteurs d'instabilité sont notés ; une évaluation est dressée et, si besoin est, des travaux de mise en ordre sont demandés par courrier spécial.

Enfin des visites ponctuelles sont effectuées sur toute demande des habitants.

\section{DÉVELOPPEMENTS APPORTÉS A LA MÉTHODOLOGIE ACTUELLE}

\subsection{Une fiche de saisie doublée d'un indice chiffré de risque}

Dans le but de donner aux opérations de saisie et d'archivage un caractère exhaustif et objectif, le repérage sur le terrain s'effectue grâce à une fiche normalisée de saisie qui permet de décrire toutes les caractéristiques physiques du terrain pouvant concerner la stabilité.

Il est joint à ce descriptif des chiffres qui expriment l'impact de chaque élément sur la stabilité de l'en- 
semble. Le total des indices renseigne sur l'appréciation globale de la propriété visitée qui peut ainsi être classée comme présentant ou non des indices suspects et comme devant ou non être surveillée, faire l'objet d'une visite complémentaire ou d'une mise en demeure d'exécuter des travaux d'urgence, etc.

\subsection{Un système informatisé d'archivage et de gestion de la prévention}

Actuellement, les informations collectées figurent dans des dossiers et dans un système de fiches cartonnées. Les données sont ainsi très dépendantes de la mémoire des agents de secteurs et sont difficiles à extraire spécifiquement. Un outil informatisé est en cours de réalisation. Il réunit les caractéristiques suivantes:

a) une base de données adresse par adresse issue des fiches de saisie normalisées avec leur complément chiffré ;

b) une base de données générales portant sur la géologie, les facteurs de risques, les éléments de la prévention, etc. ;

c) un outil de gestion de la prévention par une biblio. thèque de courriers-type en liaison avec le fichier adresse et le contenu des fiches.

Ce système ne supprime pas les dossiers, mais permet d'extraire rapidement les informations ou de rechercher localement les causes d'un incident et, surtout, de dégager du temps de présence sur le terrain en donnant un caractère quasi automatisé à un certain nombre de tâches administratives.

En effet, la pierre angulaire de la prévention municipale en matière de risques d'instabilité des versants repose sur la présence sur le terrain qui aboutit à une connaissance réciproque habitants-agents techniques et à une sensibilisation sur tous les facteurs structurels ou accidentels qui concernent ces risques.

\section{CONCLUSION}

Actuellement la prévention des risques géotechniques d'instabilité des versants des collines de la ville de Lyon s'appuie sur une cartographie des zones à risque qui permet, au sein de ces zones, de contrôler les permis de construire, d'effectuer des visites systématiques et des visites au rythme des incidents. Des actions de traitement sont également effectuées par la municipalité sur son domaine ou dans le domaine privé lorsque les habitants sont insolvables et qu'il s'agit de confortements lourds.

Cette action sera rendue plus efficace encore par la réalisation d'un outil informatisé de gestion des connaissances et des actes administratifs afférents à ces problèmes. Cette base de données comportera des informationss locales, générales et une branche fonctionnelle. Elle comporte notamment l'application d'un indice chiffré indiquant la stabilité de la propriété et permettant de faire une gestion globale des risques repérés.

\section{BIBLIOGRAPHIE}

LAREAL P., MONGEREAU N., VINET L. (1983), Balmes de Lyon: note géotechnique. Institut National des Sciences Appliquées de Lyon, décembre 1983.

LARÉAL P., MONGEREAU N., VINET L. (1988), Risque géotechnique en site urbain. Exemple de la ville de Lyon. $2^{e}$ Entretiens du Centre JacquesCartier, Montréal, $14^{e}$ Colloque.

MONGEREAU N., LARÉAL P. (1983), Bilan hydrogéologique des collines de la ville de Lyon. Exemple

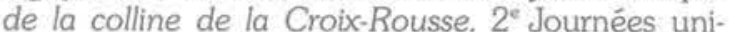
versitaires de Mécanique des Sols Appliquée, Nancy, p. 351-357.

MONGEREAU N., SANGLERAT G., DAVID L., MIL. LERS H. (1985), Mouvements de terrain en zone urbaine : exemple de la ville de Lyon. Bull. Ass. Internat. Géol. Ing., n³1, p. 95-103.

MONGEREAU N., VINET L. (1984), Balme du 13. 16, cours d'Herbourville: note géologique. INSA Lyon, octobre 1984, 8 p., 5 fig., 6 pl. photo.

RUSSO P. (1964), Géologie et hydrologie appliquées à l'urbanisme dans les collines lyonnaises. Tome 1 : La Croix-Rousse. Lyon, Audin et Cie, 201 p.

VINET L. (1991), Typologie et prévention des risques et accidents géotechniques en site urbain: les collines de la ville de Lyon entre 1977 et 1990. Thèse doctorat, INSA Lyon, 91 ISAL 62-335 p. 